\title{
THE ADVANCED COMPOSITION EXPLORER
}

E. C. Stone, L. F. Burlaga ${ }^{b}$, A. C. Cummings, ${ }^{a}$ W. C. Feldman ${ }^{c}$ W. E. Frain, J. Geiss, G. Gloeckler, R. E. Gold, D. Hovestadt, S. M. Krimigis, G. M. Mason, D. McComas, , R. A. Mewaldt, J. A. Simpson, T. T. von Rosenvinge, and M. E. Wiedenbeck ${ }^{h}$

a) California Institute of Technology; Pasadena, CA 91125 USA

b) Goddard Space Flight Center; Greenbelt, MD 20771 USA

c) Los Alamos National Laboratory; Los Alamos, NM 87545 USA

d) Physikalisches Institut, University of Bern; Bern, Switzerland

e) University of Maryland; College Park, MD 20742 USA

f) Applied Physics Lab, The Johns Hopkins University; Laurel, MD 20707 USA

g) Max-Planck-Institut fur Physik und Astrophysik; Munich, Federal Republic of Germany

h) University of Chicago; Chicago, IL 60637 USA

\begin{abstract}
The Advanced Composition Explorer (ACE) was recently selected as one of two new Explorer-class missions to be developed for launch during the mid-1990's. ACE will observe particles of solar, interplanetary, interstellar, and galactic origins, spanning the energy range from that of the solar wind $(\sim 1 \mathrm{keV} /$ nucleon) to galactic cosmic ray energies (several hundred $\mathrm{MeV} /$ nucleon). Definitive studies will be made of the abundance of nearly all isotopes from $H$ to $\mathrm{Zn}(1 \leq \mathrm{Z} \leq 30)$, with exploratory isotope studies extending to $\mathrm{Zr}(Z=40)$. To accomplish this, the ACE payload includes six high-resolution spectrometers, each designed to provide the optimum charge, mass, or charge-state resolution in its particular energy range, and each having a geometry factor optimized for the expected flux levels, so as to provide a collecting power a factor of 10 to 1000 times greater than previous or planned experiments. The payload also includes several instruments of standard design that will monitor solar wind and magnetic field conditions and energetic $\mathrm{H}$, $\mathrm{He}$, and electron fluxes. We summarize here. the scientific objectives, instrumentation, spacecraft, and mission approach that were defined for $\mathrm{ACE}$ during the Phase-A study period.
\end{abstract}

\section{INTRODUCTION}

There are a variety of energetic particle populations observed in near-Earth interplanetary space, as indicated in Figure 1. These include nuclei accelerated in the solar wind and in solar flares, in interplanetary space, in galactic cosmic ray sources, and apparently, in the case of the "anomalous" cosmic rays, at the termination shock of the solar wind. Measurements of these particles allow a direct study of the elemental and isotopic composition of three distinct samples of matter. Of these, the anomalous cosmic rays are thought to be a sample of the very local interstellar medium, while galactic cosmic rays are a second contemporary sample 


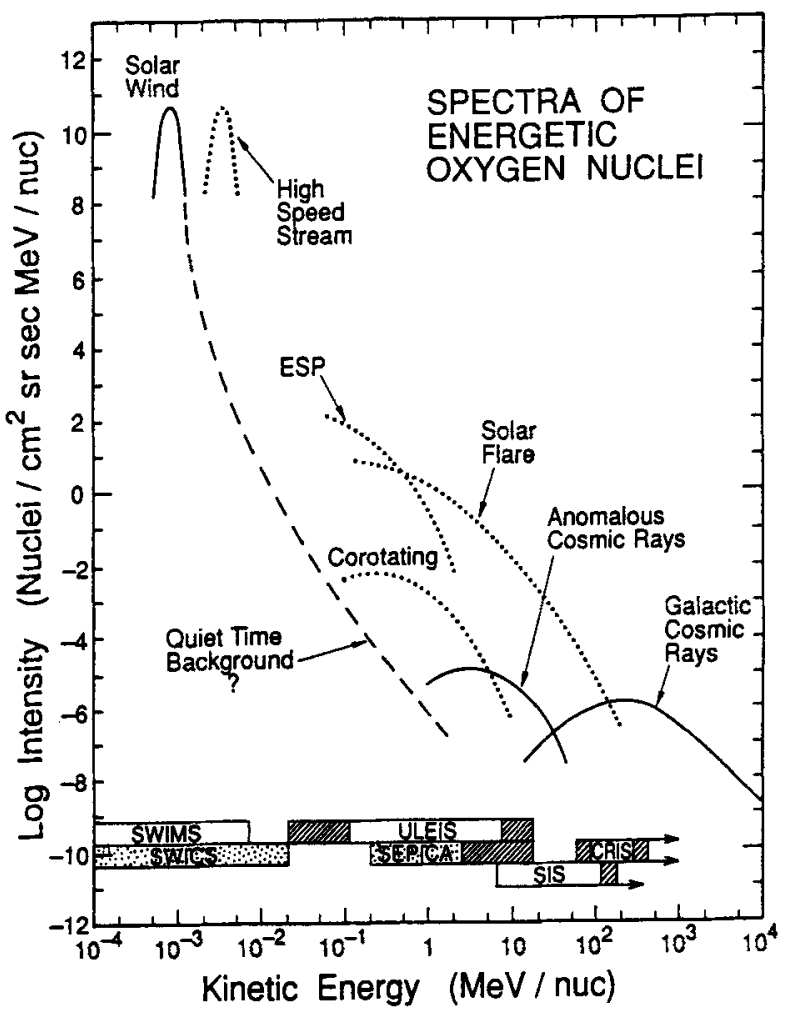

Figure 1: Typical energy spectra of energetic ${ }^{10} \mathrm{O}$ nuclei resulting from various particle populations. The solid curves represent "steady-state" components, while the dotted curves are for "transient" phenomena. Also shown is a postulated quiet-time flux of supra-thermal solar particles (dashed curve). The energy ranges of the various ACE instruments are indicated for resolution of isotopes (blank), elements only (cross-hatched), and ionic charge-states (stippled). Other nuclear species generally tend to have spectra that are similarly shaped to that of oxygen (but of varying intensity) when plotted as a function of energy/nucleon.

from somewhat more remote regions of the galaxy. The solar wind and solar energetic particles represent an older sample of interstellar matter that has been stored in the Sun for the last 4.6 billion years.

ACE will compare the composition of these distinct samples of matter by performing comprehensive and coordinated determinations of the elemental and isotopic composition of energetic nuclei accelerated on the Sun, in interplanetary space, and from galactic sources. These observations will span six decades in energy, as indicated in Figure 2, extending from solar wind to galactic cosmic ray energies, and will cover the element range from ${ }_{1} \mathrm{H}$ to ${ }_{40} \mathrm{Zr}$. The comparison of these samples of matter will be used to study the origin and subsequent evolution of both solar system and galactic material by isolating the effects of fundamental processes that include nucleosynthesis, charged and neutral-particle separation, bulk plasma acceleration, and the acceleration of suprathermal and high energy particles. 


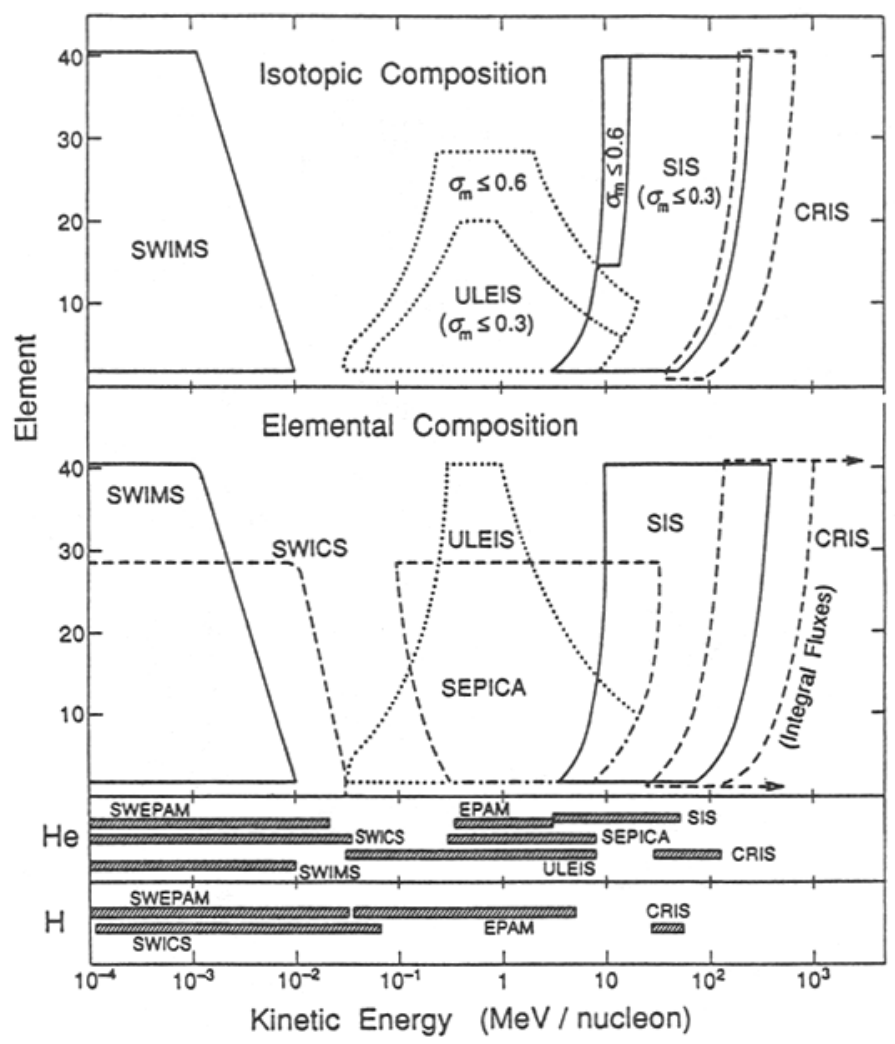

Figure 2: A plot of the energy/nucleon range over which the various ACE sensors will measure the elemental and isotopic composition of nuclei from $\mathrm{He}$ to $\mathrm{Zr}(\mathrm{Z}=2$ to 40$)$. In the top panel, boundaries for a mass resolution of $\sigma_{M} \leq 0.3$ and $\sigma_{M} \leq 0.6$ amu are indicated (note that many studies, e.g., ${ }^{22} \mathrm{Ne} /{ }^{20} \mathrm{Ne}$, do not require resolution of adjacent isotopes). The $\mathrm{H}$ and $\mathrm{He}$ panels at the bottom indicate the coverage for these elements. Note that a $400 \mathrm{~km} / \mathrm{sec}$ solar wind velocity corresponds to $\sim 8 \times 10^{-1} \mathrm{MeV} /$ nucleon.

\section{SCIENTIFIC OBJECTIVES}

The observations to be performed on $\mathrm{ACE}$ will allow the investigation of a wide range of fundamental problems in the following major areas:

1) The Elemental and Isotopic Composition of Matter

A major objective is the accurate and comprehensive determination of the elemental and isotopic composition of the various samples of "source material" from which nuclei are accelerated. Thus, using ACE measurements we will:

- Generate a set of solar isotopic abundances based on direct sampling of solar material.

- Determine the coronal elemental and isotopic composition with greatly improved accuracy. 
- Establish the pattern of isotopic differences between galactic cosmic ray and solar system matter.

- Measure the elemental and isotopic abundances of interstellar and interplanetary "pick-up ions".

- Determine the isotopic composition of the "anomalous cosmic ray component", thought to represent a sample of the local interstellar medium.

2) Origin of the Elements and Subsequent Evolutionary Processing

Isotopic "anomalies" in meteorites indicate that the solar system was not homogeneous when formed, while other data suggest that the solar composition continues to evolve. Similarly, the galaxy is neither uniform in space nor constant in time due to continuous stellar nucleosynthesis. Using measurements from ACE we will:

- Search for additional differences between the isotopic composition of solar and meteoritic material.

- Determine the contributions of solar wind and solar flare nuclei to lunar and meteoritic material, and to planetary atmospheres and magnetospheres.

- Determine the dominant nucleosynthetic processes that contribute to cosmic ray source material.

- Determine whether cosmic rays are a sample of freshly synthesized material (e.g., from supernovae), or of the contemporary interstellar medium.

- Search for isotopic patterns in solar and galactic material as a test of galactic evolution models.

3) Formation of the Solar Corona and Acceleration of the Solar Wind

Solar energetic particle, solar wind, and spectroscopic observations show that the elemental composition of the corona is differentiated from that of the photosphere, although the processes by which this occurs, and by which the solar wind is subsequently accelerated, are poorly understood. The detailed composition and charge-state data provided by ACE will allow us to:

- Isolate the dominant coronal formation processes by comparing a broad range of coronal and photospheric abundances.

- Study plasma conditions at the source of the solar wind and the solar energetic particles by measuring and comparing the charge states of these two populations.

- Study solar wind acceleration processes and any charge or mass-dependent fractionation in various types of solar wind flows.

4) Particle Acceleration and Transport in Nature

Particle acceleration is ubiquitous in nature and is one of the fundamental problems of space plasma astrophysics. The unique data set obtained by ACE measurements will enable us to: 
- Make direct measurements of charge and/or mass-dependent fractionation during solar flare and interplanetary acceleration.

- Constrain solar flare and interplanetary acceleration models with charge, mass, and spectral data spanning up to five decades in energy.

- Test theoretical models for ${ }^{3} \mathrm{He}$-rich flares and solar $\gamma$-ray events.

- Measure cosmic ray acceleration and propagation time scales using radioactive clocks.

- Test whether the "anomalous cosmic rays" are a singly-ionized sample of the neutral interstellar gas by directly measuring their charge state.

\section{INSTRUMENT SYSTEM}

The scientific objectives described above imply a need for coordinated highprecision measurements of the isotopic, elemental, and ionic charge state composition of energetic nuclei over a broad energy range, made with excellent time resolution in order to account for dynamical processes that affect the composition. The ACE sensor systems employ the optimum technology for each energy regime.

The sensor systems for the ACE payload are:

SWIMS - A Solar Wind Ion Mass Spectrometer that uses time-of-flight (TOF) through a specially tailored electric field to achieve unprecedented mass resolution at solar wind energies.

SWICS - A Solar Wind Ion Composition Spectrometer which is the Ulysses flight spare of the GLG ${ }^{1}$ instrument and which uses electrostatic deflection, postacceleration, TOF, and energy measurement to determine the elemental and ioniccharge composition of the solar wind.

ULEIS - An Ultra Low Energy Isotope Spectrometer that combines TOF with energy measurement to achieve excellent mass resolution for particles with energies $\sim 20 \mathrm{keV} /$ nuc to $10 \mathrm{MeV} /$ nuc.

SEPICA - A Solar Energetic Particle Ionic Charge Analyzer that uses electrostatic deflection, differential energy loss $(d E / d X)$, and residual energy measurement $\left(E^{\prime}\right)$ to obtain the ionic charge state and nuclear charge of energetic ions above $\sim 0.2$ $\mathrm{MeV} /$ nuc.

SIS - A Solar Isotope Spectrometer that uses trajectory measuring detectors, $\mathrm{dE} / \mathrm{dX}$, and $\mathrm{E}^{\prime}$ measurements to determine the elemental and isotopic composition of solar, anomalous, and interplanetary particles from He to $\mathrm{Zn}(\mathrm{Z}=2$ to 30$)$ over the energy range from $\sim 8$ to $150 \mathrm{MeV} /$ nuc.

CRIS - A Cosmic Ray Isotope Spectrometer that uses trajectory measurements, $\mathrm{dE} / \mathrm{dX}$, and $\mathrm{E}^{\prime}$ measurements to provide high-statistics measurements of all stable and long-lived isotopes of galactic cosmic ray nuclei from $\mathrm{He}$ to $\mathrm{Zn}(\mathrm{Z}=2$ to 30 ) over the energy range from $\sim 100$ to $600 \mathrm{MeV} / \mathrm{nuc}$.

SWEPAM - A Solar Wind Electron, Proton, and Alpha-particle Monitor which is the Ulysses flight spare of the BAM $^{2}$ instrument and which will provide baseline 


\begin{tabular}{|c|c|c|c|c|c|}
\hline \multicolumn{6}{|c|}{ Table 1: Instrument System Summary } \\
\hline Instrument & $\begin{array}{l}\text { Wt. } \\
(\mathrm{kg})\end{array}$ & $\begin{array}{l}\text { Nom. } \\
\text { Power } \\
\text { (W) }\end{array}$ & $\begin{array}{c}\text { Data } \\
\text { Rate } \\
\text { (bits/s) } \\
\end{array}$ & Technique & $\begin{array}{c}\text { Typical } \\
\text { Energy } \\
(\mathrm{MeV} / \text { nuc }) \\
\end{array}$ \\
\hline CRIS & 20.6 & 10.6 & 462 & $\mathrm{dE} / \mathrm{dX} \times \mathrm{E}$ & $\sim 300$ \\
\hline SIS & 19.5 & 18.1 & 2000 & $\mathrm{dE} / \mathrm{dX} \times \mathrm{E}$ & $\sim 30$ \\
\hline ULEIS & 17.0 & 15.1 & 1000 & TOF $\times \mathrm{E}$ & $\sim 1$ \\
\hline SEPICA & 16.0 & 6.0 & 600 & $\mathrm{dE} / \mathrm{dX} \times \mathrm{E} \times \mathrm{E} / \mathrm{Q}$ & $\sim 1$ \\
\hline SWICS & 5.5 & 4.0 & 500 & $\mathrm{TOF} \times \mathrm{E} \times \mathrm{E} / \mathrm{Q}$ & $\sim 0.001$ \\
\hline SWIMS & 6.8 & 5.0 & 505 & $\begin{array}{l}\text { TOF thru special } \\
\text { E-field }\end{array}$ & $\sim 0.001$ \\
\hline SWEPAM & 6.7 & 5.4 & 1000 & $\begin{array}{c}\text { Electrostatic } \\
\text { Analyzer }\end{array}$ & $\sim 0.001$ \\
\hline $\begin{array}{l}\text { SWIMS/SWICS/ } \\
\text { SWEPAM DPU }\end{array}$ & 3.0 & 3.0 & - & & - \\
\hline EPAM & 5.0 & 5.0 & 160 & $\mathrm{dE} / \mathrm{dX} \times \mathrm{E}$ & $\sim 0.3$ \\
\hline MAG & 4.7 & 4.0 & 300 & Triaxial fluxgate & - \\
\hline
\end{tabular}

monitoring of solar wind electron and ion fluxes.

EPAM - An energetic Electron, Proton, and Alpha-particle Monitor which is the Ulysses flight spare of the $\mathrm{LAN}^{3}$ instrument and which will provide baseline monitoring of low-energy particle fluxes.

MAG - A Magnetometer which is the Voyager Proof Test Model ${ }^{4}$ and which will provide baseline monitoring of the interplanetary magnetic field.

Table 1 provides a summary of some of the important instrument parameters.

There are previous and planned space flight instruments which have and will be providing definitive studies of the most abundant isotopes, exploratory studies ( $<100$ particles) of some of the less abundant nuclides, and surveys of the composition of the solar wind. We summarize here a comparison of the capabilities of these earlier instruments with those planned for ACE. In comparing energetic particle instruments of comparable resolution it should be remembered that for resolved isotope measurements it is the collecting power of the instrument that determines the statistical accuracy to which a given isotope ratio can be measured. It is also the collecting power that determines the minimum time scale on which the composition of transient events can be characterized. These collecting power estimates take into account all essential features of instrument design, including the geometry factor, energy range, immunity to "pile-up" and "accidental coincidences", and telemetry bit rate.

Solar Wind Ion Mass Spectrometers: As indicated in Table 2, our present knowledge of the isotopic composition of the solar wind is very limited. SWIMS will extend solar-wind spectroscopy to a wide range of additional elements and isotopes.

Solar and Interplanetary Isotope Spectrometers: Figure 3 compares the characteristics of ULEIS and SIS with other instruments designed to measure solar 


\begin{tabular}{|c|c|c|c|c|c|c|c|}
\hline \multirow{2}{*}{ Element } & \multicolumn{6}{|c|}{ Time Period of Measurements } & \multirow{2}{*}{$\begin{array}{c}\text { ACE/ } \\
\text { SWIMS } \\
\end{array}$} \\
\hline & 1960 & 1965 & 1970 & 1975 & 1980 & 1985 & \\
\hline $\begin{array}{l}\mathrm{H} \\
\mathrm{He}\end{array}$ & $\mathrm{E}$ & $\begin{array}{l}\mathrm{E} \\
\mathrm{E}\end{array}$ & $\begin{array}{c}E \\
I\end{array}$ & $\begin{array}{l}\mathrm{E} \\
\mathbf{E}\end{array}$ & $\begin{array}{c}E \\
I\end{array}$ & $\begin{array}{c}E \\
I\end{array}$ & $\begin{array}{l}\mathrm{E} \\
\mathrm{I}\end{array}$ \\
\hline $\begin{array}{l}\mathrm{C} \\
\mathrm{N} \\
\mathrm{O}\end{array}$ & & & E & $\mathrm{E}$ & $\mathrm{E}$ & $\begin{array}{l}\mathrm{E} \\
\mathrm{E} \\
\mathrm{E}\end{array}$ & $\begin{array}{l}\text { I } \\
\text { I } \\
\text { I }\end{array}$ \\
\hline $\begin{array}{l}\mathrm{Ne} \\
\mathrm{Na} \\
\mathrm{Mg}\end{array}$ & & & 1 & & $\mathrm{E}$ & $\begin{array}{l}\mathrm{E} \\
\mathrm{E}\end{array}$ & $\begin{array}{l}\text { I } \\
\text { I } \\
\text { I }\end{array}$ \\
\hline $\begin{array}{l}\text { Al } \\
\mathrm{Si} \\
\mathrm{P}\end{array}$ & & & $\mathrm{E}$ & $\mathrm{E}$ & $\mathrm{E}$ & $\mathrm{E}$ & $\begin{array}{l}\text { I } \\
\text { I } \\
1\end{array}$ \\
\hline $\begin{array}{c}\mathrm{S} \\
\mathrm{Cl} \\
\mathrm{Ar}\end{array}$ & & & 1 & & & $\mathrm{E}$ & $\begin{array}{l}\text { I } \\
\text { I } \\
\text { I }\end{array}$ \\
\hline $\begin{array}{l}\mathrm{K} \\
\mathrm{Ca} \\
\mathrm{Ti}\end{array}$ & & & & & & & $\begin{array}{l}I \\
I \\
I\end{array}$ \\
\hline $\begin{array}{c}\mathrm{V} \\
\mathrm{Cr} \\
\mathrm{Mn}\end{array}$ & & & & & & & $\begin{array}{l}\text { I } \\
\text { I } \\
\text { I }\end{array}$ \\
\hline $\begin{array}{l}\mathrm{Fe} \\
\mathrm{Co} \\
\mathrm{Ni}\end{array}$ & & & E & E & $\mathrm{E}$ & $\mathrm{E}$ & $\begin{array}{l}\text { I } \\
\text { I } \\
\text { I }\end{array}$ \\
\hline $\begin{array}{l}\text { Technique } \\
\text { Used: }\end{array}$ & $\begin{array}{l}\text { Faraday } \\
\text { Cup (F.C.) }\end{array}$ & $\begin{array}{l}\text { F.C., } \\
\text { E/Q }\end{array}$ & $\begin{array}{l}\text { E/Q } \\
\text { Foil }\end{array}$ & $\mathrm{E} / \mathrm{Q}$ & $M / Q$ & $\begin{array}{l}\text { E/Q-A } \\
\text { TOF-E }\end{array}$ & $\begin{array}{l}\text { E/Q-TOF } \\
V=X^{2}\end{array}$ \\
\hline
\end{tabular}

E: Indicated elements or element groups are resolved.

I: Listed isotopes of indicated elements are resolved.

and interplanetary isotopes with a mass resolution of $\leq 0.25$ amu at Si. ULEIS will provide the first comprehensive isotope measurements below a few $\mathrm{MeV} / \mathrm{nuc}$.

Solar Energetic Particle Charge Analyzers: Through the use of increased electrostatic deflection voltages and the addition of a time-of-flight system with position sensing, SEPICA will achieve a factor of $\sim 25$ increase in collecting power over previous or planned instruments. At the same time, the charge-state resolution near $1 \mathrm{MeV} / \mathrm{nuc}$ will be greatly improved.

Galactic Cosmic Ray Spectrometers: Figure 4 compares the proposed SIS and CRIS instruments with those of other investigations. Note that CRIS will provide the necessary order of magnitude increase in collecting power to provide definitive measurements of rare isotopes, while SIS will extend these measurements to lower energy, also with very large collecting power. Although a balloon-borne instrument may have 5 to 10 times the collecting power of CRIS, it would have a duty cycle of $<10^{-2}$ (i.e., 2 days/yr). The superconducting magnet facility planned for the Space Station (Astromag) will be limited to energies $>2 \mathrm{GeV} / \mathrm{nuc}$ by the geomagnetic cutoff. Thus, ACE and Astromag observations will complement each other. 

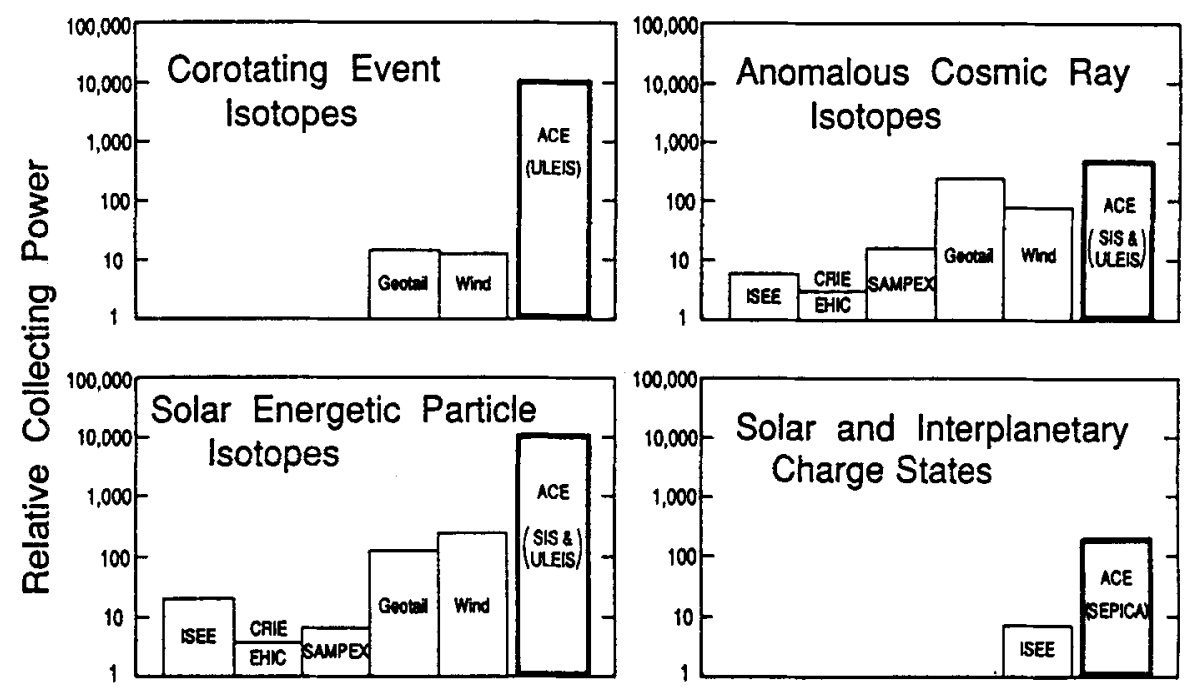

Figure 3: A comparison of the collecting power of previous and planned spectrometers designed to measure the isotopic composition and charge states of solar and interplanetary particles. Note that the collecting power scale is logarithmic.

\section{SPACECRAFT, MISSION, and DATA ANALYSIS APPROACH}

To minimize cost, the proposed ACE spacecraft is based on designs that evolved from the AMPTE/CCE and SIMPL programs. The AMPTE/CCE spacecraft was designed as a Sun-pointing mounting bus for scientific instruments, built by The Johns Hopkins University/Applied Physics Laboratory, and successfully launched in August 1984. The SIMPL study defined a spacecraft at the Earth/Sun $L_{1}$ point that would provide an early warning for solar-terrestrial disturbances.

The ACE spacecraft bus provides mounting platforms, electrical power, attitude control, thermal control, and data handling for the 9 scientific instruments. The spacecraft body consists of a two deck, irregular octagon, 78 inches across the points and about 4 feet high. Most of the instruments and spacecraft electronics are mounted on the upper deck. The lower deck supports a hydrazine propulsion system that provides for both orbit insertion and maintenance. Electrical power is supplied by four solar panels and an $8 \mathrm{~A}$-hr battery. RF communication is achieved with a $60-\mathrm{cm}$ high-gain, parabolic dish antenna and four low-gain quadrifilar helical antennas.

A Multi-purpose Launch Vehicle (MLV) with the capability of a Delta II 7920 will deliver the ACE spacecraft to the vicinity of the Earth-Sun libration point, $L_{1}$. The spacecraft propulsion system will maneuver ACE so that an orbit of 10 degrees with respect to an Earth-Sun line will be established. Periodic thrust maneuvers and spin axis torquing will be required over the mission life. The 


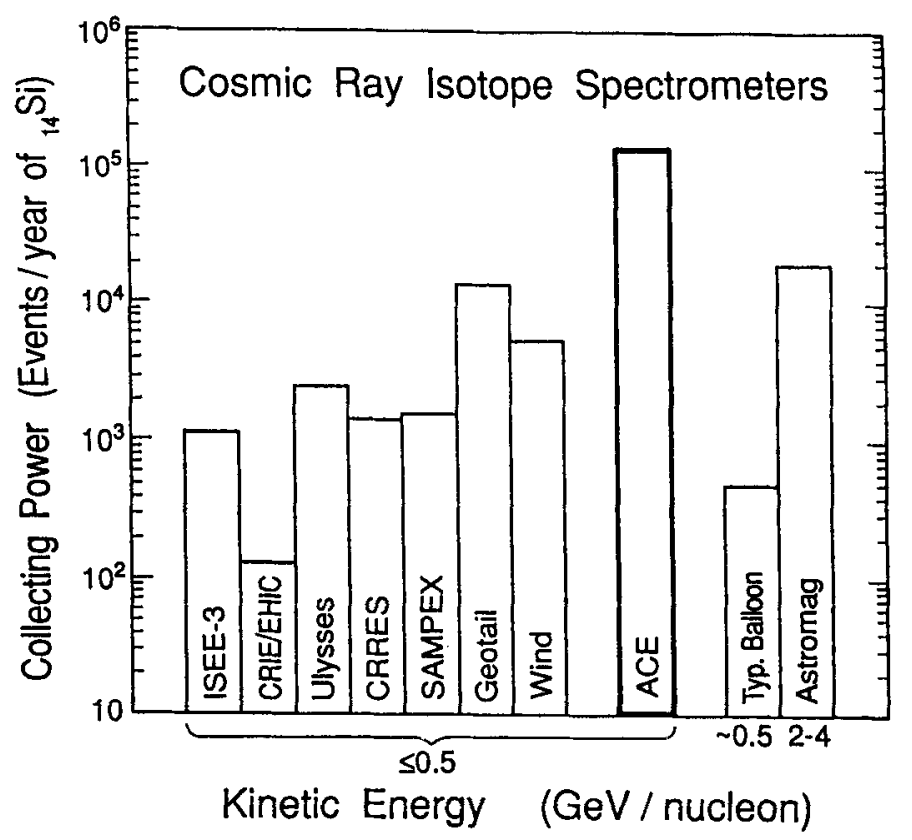

Figure 4: A comparison of the collecting power of previous and planned cosmic ray isotope spectrometers with that of $\mathrm{ACE}$.

spacecraft attitude will be set to provide spin axis pointing toward the Sun to within $\pm 20^{\circ}$. Data will be collected on tape and will be played back once per day to the 26-meter antennas of the Deep Space Network. The data are then transmitted to the ACE ground station for analysis and subsequent distribution.

Correlation of the diverse measurements is a key component of the $\mathrm{ACE}$ science objectives. Consequently, the initial science processing of the telemetry stream will be accomplished at a single central Production Data Center (PDC), which will convert the data to calibrated science measurements. This data set will be made available to nine Analysis Data Sites (ADS), located at each of the local Investigator Institutions, where comprehensive local science analysis will take place. In addition, each Investigator Team will supply a set of algorithms that will enable the other Investigators to display summary data in a standard format to facilitate correlation amongst the various data sets. A subset of the calibrated science measurements will be made available to the National Space Science Data Center in parallel. Finally, ACE has the capability to transmit in real time a set of monitoring data to provide a continuous monitor of solar and interplanetary conditions.

\section{SUMMARY}

In summary, ACE will use advanced spectrometers with large collecting power and unprecedented resolution to make possible a major advance in our knowledge 
of the elemental and isotopic composition of solar, interplanetary, and interstellar particles over a wide energy range. The ACE data plan will allow correlations of the various data sets in order to address outstanding scientific problems in the areas of the origin of the elements, the formation of the solar corona, and particle acceleration and transport in nature.

\section{ACKNOWLEDGEMENTS}

The ACE Phase-A study could not have been completed without the contributions of many individuals, including exceptional efforts from many scientists, engineers, and staff at the various Investigator Institutions. D. Mehoke of JHU/APL was the systems engineer for the spacecraft study team. He and the other members of the spacecraft study team at JHU/APL deserve thanks for their response to the tasks given them. We are grateful to R. Farquhar of GSFC and D. Dunham of Computer Sciences Corp. for providing trajectory calculations for ACE. The ACE Technical Officers at GFSC were E. Mercanti and T. Karras and their aid in providing liaison with the GSFC Project Office was appreciated. We are thankful for the support of S. Nylund of JHU/APL who was largely responsible for formulating the ACE Data Plan. We also appreciate the assistance of G. Floyd of GSFC for helping to formulate the ground data capture and mission operations and control systems. Our efforts in defining the telecom parameters for ACE benefited from discussions with W. L. Martin of JPL. Finally, the ACE Phase-A report achieved its final form due to the considerable effort and expertise of $\mathrm{L}$. Sartain, C. Silva, and K. Gary, all of Caltech. The ACE Phase-A Study was supported by NASA Contract NAS5-30340.

\section{REFERENCES}

1. Gloeckler, G., J. Geiss, H. Balsiger, L. A. Fisk, F. Gliem, F. M. Ipavich, K. W. Ogilvie, W. Stüdemann, and B. Wilken, ESA Special Publication SP1050, 77 (1983).

2. Bame, S. J., J. P. Glore, D. J. McComas, K. R. Moore, J. C. Chavez, T, J. Ellis, G. R. Peterson, J. H. Temple, and F. J. Wymer, ESA Special Publication SP-1050, 49 (1983).

3. Lanzerotti, L. J., R. E. Gold, L. A. Anderson, T. P. Armstrong, R. P. Lin, S. M. Krimigis, M. Pick, E. C. Roelof, E. T. Sarris, G. M. Simnett, and W. E. Frain, ESA Special Publication SP-1050, 143 (1983).

4. Behannon, K. W., M. H. Acuña, L. F. Burlaga, R. P. Lepping, N. F. Ness, and F. M. Neubauer, Space Sci. Rev., 21, 235, (1977). 